\title{
Spaces with a Finite Family of Basic Functions*
}

\author{
Paul Gartside ${ }^{\dagger}$ and Ziqin Feng ${ }^{\ddagger}$ \\ Dedicated to Bob Heath on his retirement
}

July 2008

\begin{abstract}
A $T_{1}$ completely regular space $X$ is finite dimensional, locally compact and separable metrizable if and only if $X$ has a finite basic family: functions $\Phi_{1}, \ldots, \Phi_{n}$ such that for all $f \in C(X)$ there are $g_{1}, \ldots, g_{n} \in C(\mathbb{R})$ satisfying $f(x)=\sum_{i=1}^{n} g_{i}\left(\Phi_{i}(x)\right)$ for all $x \in X$.

This give the complete solution to four problems on basic functions posed by Sternfeld.
\end{abstract}

\section{Introduction}

The 13th Problem of Hilbert's celebrated list [3] is usually interpreted as asking whether every continuous real valued function of three variables can be written as a superposition (i.e. composition) of continuous functions of two variables. Kolmogorov gave a strong positive solution (we write $C(X)$ for all continuous real valued maps on a topological space $X$, and $C^{*}(X)$ for the subset of bounded maps):

Theorem 1 (Kolmogorov Superposition, [6]) For a fixed $n \geq 2$, there are $n(2 n+1)$ maps $\phi_{p q} \in C([0,1])$ such that every map $f \in C\left([0,1]^{n}\right)$ can be written:

$$
f(\mathbf{x})=\sum_{q=1}^{2 n+1} g_{q}\left(\Phi_{q}(\mathbf{x})\right) \quad \text { where } \Phi_{q}\left(x_{1}, \ldots, x_{n}\right)=\sum_{p=1}^{n} \phi_{p q}\left(x_{p}\right),
$$

and the $g_{q} \in C(\mathbb{R})$ are maps depending on $f$.

In addition to solving the superposition problem, Kolmogorov's theorem says that the functions $\Phi_{1}, \ldots, \Phi_{2 n+1}$ from $[0,1]^{n}$ to the reals form a finite 'basis' for all continuous real valued maps from $[0,1]^{n}$. This is a very striking phenomena, leading to the following natural definition of Sternfeld [11:

\footnotetext{
*2000 Mathematics Subject Classification: 26B40, 54C30; 54C35, 54E45.

Key Words and Phrases: Superposition of functions , finite dimension, locally compact, basic family, Hilbert's 13th Problem.

${ }^{\dagger}$ Corresponding author Department of Mathematics, University of Pittsburgh, Pittsburgh, PA 15260, USA, email: gartside@math.pitt.edu.

$\ddagger$ Department of Mathematics, University of Pittsburgh, PA 15260, USA
} 
Definition 2 Let $X$ be a topological space. A family $\mathbf{\Phi} \subseteq C(X)$ is said to be basic (respectively, basic ${ }^{*}$ ) for $X$ if each $f \in C(X)$ (respectively, $C^{*}(X)$ ) can be written: $f=\sum_{q=1}^{n}\left(g_{q} \circ \Phi_{q}\right)$,

for some $\Phi_{1}, \ldots, \Phi_{n}$ in $\boldsymbol{\Phi}$ and 'co-ordinate functions' $g_{1}, \ldots, g_{n} \in C(\mathbb{R})$.

Beyond their intrinsic interest, basic functions have proved to be widely useful. Since the use of basic functions reduces calculations of functions simply to addition and evaluation of a fixed finite family of functions, applications to numerical analysis, approximation and function reconstruction are immediately apparent. But other applications have emerged including to neural networks [4, 5, 7].

Extending the Kolmogorov Superposition Theorem, Ostrand 9] showed that every compact metric space of dimension $n$ has a basic family of size $2 n+1$. Subsequently Sternfeld [11 showed that this basic family is minimal in the sense that a compact metric space with a basic family of size no more than $2 n+1$ must have dimension $\leq n$. Noting that Doss [1] had shown that Euclidean $n$-space, $\mathbb{R}^{n}$, has a basic family of size $4 n$ for $n \geq 2$, Sternfeld asked (in Problems 9-13 of [11) exactly which spaces have a finite basic family, and whether the minimal size of a basic family on a space $X$ was $2 n+1$ where $n=\operatorname{dim}(X)$. Hattori [2] showed that every locally compact, separable metrizable space $X$ of dimension $n$ has a finite basic* family of size $2 n+1$. He asked whether the restriction to locally compact spaces was necessary. Our Main Theorem below gives a strong and complete solution to all these problems.

Since spaces with a finite basic family are finite dimensional, it seems plausible that spaces with a countable basic family would be countable dimensional. But we prove that if a space has a countable basic family, then some finite subcollection is also basic, and so the space is finite dimensional (and locally compact, separable metrizable). To facilitate the proof, and provide full generality we make the following definition allowing more general superposition representations than a 'basic' representation.

Definition 3 Let $X$ be a topological space. A family $\mathbf{\Phi} \subseteq C(X)$ is said to be generating (respectively, generating*) for $X$ with respect to a 'set of operations' $M$ of continuous functions mapping from subsets of Euclidean space into subsets of Euclidean space, if each $f \in C(X)$ (respectively, $C^{*}(X)$ ) can be written as a composition of functions from $\boldsymbol{\Phi}, M$ and $C(\mathbb{R})$.

Note that a basic (respectively, basic*) family is generating (respectively, generating*) with respect to $M=\{+\}$, and clearly 'generating' implies 'generating*'.

Theorem 4 (Main Theorem) Let $X$ be $T_{1}$ and completely regular. Then the following are equivalent:

1) $X$ has a countable generating ${ }^{*}$ family with respect to a countable set of operations,

2) $X$ has a finite basic family, and

3) $X$ is a finite dimensional, locally compact and separable metrizable. 
Further, a locally compact separable metrizable space $X$ has dimension $\leq n$ if and only if it has a basic family of size $\leq 2 n+1$.

By the preceding note, 2) $\Longrightarrow 1$ ) is immediate. In the next section (Section 2) we prove 1) $\Longrightarrow 3$ ), in Section 3 we establish 3 ) $\Longrightarrow 2$ ), and we justify the 'Further' claim characterizing dimension in Section 4 ,

\section{Restrictions Induced by Generating* Families}

In this section, all topological spaces are $T_{1}$ and completely regular.

Lemma 5 Let $X$ have a generating* family $\mathbf{\Phi}$ with respect to $M$. Then e : $X \rightarrow \mathbb{R}^{\boldsymbol{\Phi}}$ defined by $e(x)=(\Phi(x))_{\Phi \in \boldsymbol{\Phi}}$ is an embedding.

Proof. Clearly $e$ is continuous (each projection is a $\Phi$ in $\boldsymbol{\Phi}$ which is continuous). It is also easy to see $e$ is injective. Take distinct $x, x^{\prime}$ in $X$. Pick $f \in C^{*}(X)$ such that $f(x)=0, f\left(x^{\prime}\right)=1$. Represent $f$ as a composition of $\Phi_{1}, \ldots, \Phi_{n}$ in $\boldsymbol{\Phi}$, members of $M$ and $C(\mathbb{R})$. If $e(x)=e\left(x^{\prime}\right)$ then $\Phi_{i}(x)=\Phi_{i}\left(x^{\prime}\right)$ for all $i$, and so $f(x)=f\left(x^{\prime}\right)$, which is a contradiction.

It remains to show that the topology induced on $X$ by $e$ contains the original topology. Since $X$ is completely regular it is sufficient to check that for every $f \in C^{*}(X)$ the map $e(f): e(X) \rightarrow \mathbb{R}$ defined by $e(f)(\mathbf{x})=f\left(e^{-1}(\mathbf{x})\right)$ is continuous. But each $f \in C^{*}(X)$ can be written as a composition of some $\Phi_{1}, \ldots, \Phi_{n}$ in $\boldsymbol{\Phi}$ and members of $M$ and $C(\mathbb{R})$. Note that for each $i$ we have $\Phi\left(e^{-1}(\mathbf{x})\right)=\pi_{\Phi_{i}}(\mathbf{x})$, where $\pi_{\Phi_{i}}$ is the projection map of $\mathbb{R}^{\mathbf{\Phi}}$ onto the $\Phi_{i}$ th coordinate. Hence $e(f)=f \circ e^{-1}$ is the composition of continuous maps, namely the $\pi_{\Phi_{i}}$ s and functions in $M$ and $C(\mathbb{R})$, and so is continuous as required.

Since any subspace of $\mathbb{R}^{\mathbb{N}}$ is separable metrizable, and any subspace of $\mathbb{R}^{n}$ is finite dimensional we deduce from Lemma 5 .

\section{Corollary 6}

a) A space with a countable generating* family is separable metrizable.

b) A space with a finite generating* family is finite dimensional.

A subspace $C$ of a space $X$ is said to be $C^{*}$-embedded in $X$ if every $f \in$ $C^{*}(C)$ can be extended to a continuous bounded real valued function on $X$. In a normal space all closed subspaces are $C^{*}$-embedded. Compact subspaces are always $C^{*}$-embedded. We note the following easy lemma:

Lemma 7 If $\boldsymbol{\Phi}$ is a generating ${ }^{*}$ family (respectively, basic*) for a space $X$ with respect to $M$, and $C$ is $C^{*}$-embedded in $X$ then $\boldsymbol{\Phi} \mid C=\{\Phi \mid C: \Phi \in \mathbf{\Phi}\}$ is a generating* (respectively, basic ${ }^{*}$ ) family for $C$.

Lemma 8 A space with a countable generating* family is locally compact. 
Proof. Suppose the space $X$ has a countable generating* family $\mathbf{\Phi}$ with respect to $M$, but is not locally compact. Since $X$ is metrizable, it follows that the metric fan $F$ (defined below) embeds as a closed subspace in $X$. Hence by Lemma 7 it suffices to show that $F$ does not admit a countable generating* family (with respect to any set of operations $M$ ).

The metric fan $F$ has underlying set $\{*\} \cup(\mathbb{N} \times \mathbb{N})$ and topology in which all points other than $*$ are isolated and $*$ has basic neighborhoods $B(*, N)=$ $\{*\} \cup([N, \infty) \times \mathbb{N})$. For a contradiction, let $\Phi=\left\{\Phi_{1}, \Phi_{2}, \ldots\right\}$ be a countable generating* family with respect to $M$.

For each $i$, let $y_{i}=\Phi_{i}\left(x_{0}\right)$, and pick basic open $U_{i}$ containing $*$ such that $\Phi_{i}\left(U_{i}\right) \subseteq\left(y_{1}-1, y_{1}+1\right)$. Now for each $n$ let $V_{n}=\bigcap_{i=1}^{n} U_{i}$. So $\Phi_{i}\left(V_{n}\right) \subseteq$ $\left(y_{i}-1, y_{i}+1\right)$ for $i=1, \ldots, n$. We can write $V_{n}=\{*\} \cup\left(\left[N_{n}, \infty\right) \times \mathbb{N}\right)$ and suppose, without loss of generality, that $N_{n}>N_{m}$ if $n>m$.

Fix $n$. Let $D^{0}=\left\{x_{k}^{0}=\left(N_{n}, k\right): k \in \mathbb{N}\right\}$. As $\left\{\Phi_{1}\left(x_{k}^{0}\right)\right\}_{k \in \mathbb{N}}$ is a subset of $\left[y_{1}-1, y_{1}+1\right]$, which is sequentially compact, there is a $D^{1}=\left\{x_{k}^{1}: k \in \mathbb{N}\right\} \subseteq D^{0}$ such that $\left\{\Phi_{1}\left(x_{k}^{1}\right)\right\}_{k \in \mathbb{N}}$ is convergent. As $\left\{\Phi_{2}\left(x_{k}^{1}\right)\right\}_{k \in \mathbb{N}}$ is a subset of $\left[y_{2}-1, y_{2}+\right.$ 1], which is sequentially compact, there is a $D^{2}=\left\{x_{n}^{2}: n \in \mathbb{N}\right\} \subseteq D^{0}$ such that $\left\{\Phi_{2}\left(x_{k}^{2}\right)\right\}_{k \in \mathbb{N}}$ is convergent. Inductively we get $D^{n}=\left\{x_{k}^{n}: k \in \mathbb{N}\right\}$, which is infinite closed discrete and for each $i=1, \ldots, n$ the sequence $\left\{\Phi_{i}\left(x_{k}^{n}\right)\right\}_{k \in \mathbb{N}}$ is convergent, say to $z_{i}^{n}$. Define $D_{O}^{n}=\left\{x_{2 k-1}^{n}: k \in \mathbb{N}\right\}$ and $D_{E}^{n}=\left\{x_{2 k}^{n}: k \in \mathbb{N}\right\}$.

Define $f: F \rightarrow[0,1]$ by: $f$ is identically zero outside $\bigcup_{n} D_{O}^{n}$ (in particular, $f$ is zero on each $D_{E}^{n}$ ), and $f$ is identically $1 / n$ on $D_{O}^{n}$. Then $f$ is continuous and bounded.

Hence, for some $m, f$ can be written as the composition of $\Phi_{1}, \ldots, \Phi_{m}$ and members of $M$ and $C(\mathbb{R})$. Now, on the one hand $\lim _{k} \Phi_{i}\left(x_{2 k-1}^{m}\right)=z_{i . m}=$ $\lim _{k} \Phi_{i}\left(x_{2 k}^{m}\right)$ so by continuity of the elements of $M$ and $C(\mathbb{R})$ in the compositional representation of $f, \lim _{k} f\left(x_{2 k-1}^{m}\right)=\lim _{k} f\left(x_{2 k}^{m}\right)$, and on the other hand, $\lim _{k} f\left(x_{2 k-1}^{m}\right)=1 / m \neq 0=\lim _{k} f\left(x_{2 k}^{m}\right)$. This is our desired contradiction.

Let $Y$ be a locally compact separable metrizable space. Write $C_{k}(Y)$ for $C(Y)$ with the compact-open topology. Then $C_{k}(Y)$ is a Polish (separable, completely metrizable) group. In particular, for any $n, C_{k}(\mathbb{R})^{n}$ is a Polish group.

Lemma 9 If $X$ has a countable generating* family with respect to a countable set of operations, $M$, then $X$ has a finite generating* family with respect to a finite set of operations $M^{\prime}$.

Proof. Let $\Phi_{1}, \Phi_{2}, \ldots$ be a countable generating* family for $X$ with respect to the countable set of operations $M$. By Lemma $8 X$ is locally compact and $C_{k}(X)$ is a Polish group.

Let $g_{1}, g_{2}, \ldots$ be formal letters representing functions from $\mathbb{R}$ to $\mathbb{R}$. Let $\mathcal{W}$ be the set of all formal compositions of $\Phi_{i} \mathrm{~s}$, elements of $M$ and $g_{i}$ s. Note that $\mathcal{W}$ is countable.

Fix $w$ in $\mathcal{W}$. Then $w$ induces a map $\left(g_{1}, \ldots, g_{n}\right) \mapsto w\left(g_{1}, \ldots, g_{n}\right)$ from $C_{k}(\mathbb{R})^{n} \rightarrow C_{k}(X)$ where we substitute actual $g_{i} \in C(\mathbb{R})$ for the corresponding 
formal letter. This map is continuous with respect to the compact-open topology. Let $F_{w}=w\left(C_{k}(\mathbb{R})^{n}\right)$. It is analytic. Define $G_{w}=F_{w} \cap C_{k}(X,(0,1))$. Since $C_{k}(X,(0,1))$ is homeomorphic to $C_{k}(X)$ it is Polish, and hence must be a $G_{\delta}$ subset of $C_{k}(X)$. So $G_{w}$ is analytic in $C_{k}(X,(0,1))$.

Note, by the generating* property, that $C_{k}^{*}(X) \subseteq \bigcup_{w \in \mathcal{W}} F_{w}$. Hence $C_{k}(X,(0,1))=$ $\bigcup_{w \in \mathcal{W}} G_{w}$. By the Baire Category theorem there must be some particular $w$ in $\mathcal{W}$ such that $G_{w}$ is not meager.

Fix a homeomorphism $h: \mathbb{R} \rightarrow(0,1)$. Via $h$, addition and subtraction on $\mathbb{R}$ induce (continuous) group operations $\oplus, \ominus:(0,1) \times(0,1) \rightarrow(0,1)$. These operations on $(0,1)$ in turn induce operations on $C_{k}(X,(0,1))$ making this space a Polish group.

Let $H_{w}$ be the subgroup of $C_{k}(X,(0,1))$ generated by $G_{w}$. By Pettis' Theorem [10], since $G_{w}$ is non-meager and analytic, $G_{w} \ominus G_{w}$ has non-empty interior. Hence the subgroup $H_{w}$ is open, and so coincides with $C_{k}(X,(0,1)$ ) (which is connected).

Set $\boldsymbol{\Phi}^{\prime}$ to be the finite set of $\Phi_{i}$ s appearing in $w$, and set $M^{\prime}$ to be $\oplus, \ominus$ and the finite set of elements of $M$ appearing in $w$. Since $H_{w}=C(X,(0,1))$, each element of $C(X,(0,1))$ is a composition of members of $\boldsymbol{\Phi}^{\prime}, M^{\prime}$ and $C(\mathbb{R})$.

We check $\boldsymbol{\Phi}^{\prime}$ is a finite generating* family with respect to $M^{\prime}$. For if $f \in$ $C^{*}(X)$, then $f$ maps into some open interval $(a, b)$. Fix a homeomorphism $g_{0}: \mathbb{R} \rightarrow \mathbb{R}$ taking $(0,1)$ to $(a, b)$. Then $f=g_{0} \circ\left(g_{0}^{-1} \circ f\right)$, where $g_{0}^{-1} \circ f$ is in $C_{k}(X,(0,1))$. Hence $g_{0}^{-1} \circ f$ can be expressed as a composition of elements of $\boldsymbol{\Phi}^{\prime}, M^{\prime}$ and some $g_{1}, \ldots, g_{n}$ in $C(\mathbb{R})$. But now $f$ is $g_{0}$ of this composition and so is also expressible in terms of elements of $\boldsymbol{\Phi}^{\prime}, M^{\prime}$ and $C(\mathbb{R})$, as required.

We note that the finite generating* family is a subset of the original family, and also that if the original family is generating then we can take $M^{\prime} \subseteq M \cup$ $\{+,-\}$.

Proof of 1) $\Longrightarrow 3$ ).

Let $X$ be a space with a countable generating* family with respect to a countable set of operations. By Corollary [6 a) $X$ is separable metrizable. Lemma 8 then says that $X$ is locally compact. From Lemma 9 we deduce that $X$ has a finite generating* family. Hence by Corollary 6 b) $X$ is finite dimensional.

\section{Construction of Finite Basic Families}

This section is devoted to proving:

Lemma 10 If $X$ is a locally compact, separable metrizable space of dimensiosn $\leq n$ then $X$ has a basic family of size $2 n+1$.

Lemma 10 is the forward implication of the 'Further' claim in the Main Theorem. The implication '3) $\Longrightarrow 2$ )' of the Main Theorem then follows.

Recall that Hattori 2] showed that every locally compact, separable metrizable space $X$ of dimension $n$ has a finite basic* family of size $2 n+1$. Lemma 10 
and its proof improves on Hattori's result and proof because: (1) it applies to all functions (not necessarily bounded), (2) it is constructive (Hattori's argument uses a Baire category argument) and (3) it is considerably shorter than Hattori's. The proof is similar to that of Ostrand for compact metric spaces. However difficulties arise because continuous real valued functions on a locally compact space need not be bounded.

For this section, fix a locally compact, separable space $X$ of dimension $\leq n$, and with compatible metric $d$. We can find $\left\{K_{m}: m \geq-1\right\}$ a countable cover of $X$ by compact sets such that $K_{-1}=K_{0}=\emptyset$ and $K_{m} \subseteq K_{m+1}^{0}$ for each $m \geq-1$. For each $m \geq 0$ we put $H_{m}=K_{m} \backslash K_{m-1}^{\circ}$, and set $U_{m}=K_{m+1}^{\circ} \backslash K_{m-1}$. Since Ostrand has done the compact case, we can assume that the $K_{m}$ 's are strictly increasing. We show $X$ has a basic family of size $2 n+1$.

The basic functions $\Phi_{i}$ are defined to be the limit of approximations $f_{k}^{i}$. The approximations are defined inductively along with some families of 'nice' covers. These 'nice' covers come from Ostrand's [9] characterization of dimension:

Theorem 11 (Ostrand's Covering Theorem) A metric space $Y$ of dimension $\leq n$ if and only if for each open cover $\mathcal{C}$ of $Y$ and each integer $k \geq n+1$ there exist $k$ discrete families of open sets $\mathcal{U}_{1}, \ldots, \mathcal{U}_{k}$ such that the union of any $n+1$ of the $\mathcal{U}_{i}$ is a cover of $Y$ which refines $\mathcal{C}$.

Lemma 12 Let $\gamma>0$. There are $2 n+1$ many families $\mathcal{S}^{1}, \ldots, \mathcal{S}^{2 n+1}$ of open subsets of $X$, and $\eta^{m}>0$ for $m \geq 0$, satisfying:

(1) Each $\mathcal{S}^{i}$ is discrete in $X$.

(2) For $k$ fixed and each $x \in X$ fixed, $\left|\left\{S \in \bigcup_{i=1}^{2 n+1} \mathcal{S}^{i}: x \in S\right\}\right| \geq n+1$.

(3) $\operatorname{diam} S<\gamma$ for any $S \in \bigcup_{i=1}^{2 n+1} \mathcal{S}^{i}$.

(4) $\bigcup_{i=1}^{2 n+1} \mathcal{S}^{i}$ refines $\left\{U_{m}: m \in \omega\right\}$.

(5) For any $m \in \mathbb{N},\left\{S: S \in \bigcup_{i=1}^{2 n+1} \mathcal{S}^{i}, S \cap K_{m} \neq \emptyset\right\}$ is finite.

(6) $S\left(H_{m}, \eta^{m}\right) \cap S=\emptyset$ if $H_{m} \cap \bar{S}=\emptyset$ for any $S \in \bigcup_{i=1}^{n+1} \mathcal{S}^{i}$.

(7) $\overline{S\left(H_{m-1}, \eta^{m-1}\right)} \cap \overline{S\left(H_{m+1}, \eta^{m+1}\right)}=\emptyset$.

In (6) and (7), $S\left(H_{m}, \eta^{m}\right)=\left\{x \in X: d\left(H_{m}, x\right) \leq \eta^{m}\right\}$

Proof. Let $\mathcal{C}=\left\{C_{a}: a \in \mathbb{N}\right\}$ be a locally finite open cover of $X$ with: $\operatorname{diam}\left(C_{a}\right)<\gamma$ and $\left|\left\{H_{m}: H_{m} \cap \overline{C_{a}} \neq \emptyset\right\}\right| \leq 2$, for each $a \in \mathbb{N}$. Then by Ostrand's covering theorem, there exist $2 n+1$ discrete families of open sets $\mathcal{S}_{1}, \cdots, \mathcal{S}_{2 n+1}$ which refines $\mathcal{C}$. Also the union of any $n+1$ of the $\mathcal{S}_{i}$ is a cover of $X$. So 1)-4) are easy to verify.

Fix $i$ with $1 \leq i \leq 2 n+1$. As $\mathcal{S}^{i}$ is discrete, $\left\{S: S \cap K_{m} \neq \emptyset, S \in \mathcal{S}^{i}\right\}$ is finite. Thus condition 5 ) is satisfied.

Now fix $i$ and $m$, the discreteness of $\mathcal{S}^{i}$ guarantees that

$$
H_{m} \cap \overline{\bigcup\left\{S: S \in \mathcal{S}^{i} \text { and } H_{m} \cap \bar{S}=\emptyset\right\}}=\emptyset .
$$

So $d\left(H_{m}, \overline{\left.\bigcup\left\{S: S \in \mathcal{S}^{i} \text { and } H_{m} \cap \overline{(} S\right)=\emptyset\right\}}>0\right.$. Then we can pick $\eta_{i}^{m}$ such that $S\left(H_{m}, \eta_{i}^{m}\right) \cap S=\emptyset$ if $H_{m} \cap \bar{S}=\emptyset$ for any $S \in \mathcal{S}^{i}$. Let $\eta^{m}=\min \left\{\eta_{i}^{m}: i=\right.$ $1, \cdots, 2 n+1\}$. This satisfies 6$)$. 
Notice that since $H_{m}$ is compact for each $m \in \mathbb{N}$, we can pick $\eta^{m}$ small enough such that $\overline{S\left(H_{m-1}, \eta^{m-1}\right)} \cap \overline{S\left(H_{m+1}, \eta^{m+1}\right)}=\emptyset$, giving (7).

Step 1: Construction of the approximations Again, we generalize the construction of Ostrand, but must find ways around the problem of not having bounded functions.

By induction on $k \geq 0$, using Lemma 12, for $i=1, \ldots, 2 n+1$, there exist: positive real numbers $\epsilon_{k}$ with $\epsilon_{1}<1 / 4, \gamma_{k}, \eta_{k}^{m}$ distinct positive prime numbers $r_{k}^{i}$, discrete families $\mathcal{S}_{k}^{1}, \ldots, \mathcal{S}_{k}^{2 n+1}$ and continuous functions $f_{k}^{i}: X \rightarrow[0, k+1]$, with the following properties.

For each $k \in \mathbb{N}$, the families $\mathcal{S}_{k}^{1}, \ldots, \mathcal{S}_{k}^{2 n+1}, \gamma_{k}$ and $\eta_{k}^{m}$ satisfy (1)-(7) of Lemma 12, Further:

(A) $\lim _{k \rightarrow \infty} \gamma_{k}=\lim _{k \rightarrow \infty} \epsilon_{k}=0$;

(B) $\epsilon_{k}<1 / \Pi_{i=1}^{2 n+1} r_{k}^{i}$;

(C) $f_{k}^{i}$ is constant on the closure of those members of $\mathcal{S}_{k}^{i}$ which have nonempty intersection with $K_{m}$ for $(m \leq k)$, the constant being an integral multiple of $1 / r_{k}^{i}$, and takes different values on distinct members. Then we can take a continuous extension of $f_{k}^{i}$ to the rest of the space.

(D) For any $S$ in $\mathcal{S}_{k}^{i}$ having nonempty intersection with $H_{m}, m-1<f_{m}^{i}(S)<$ $m+1$. Also for $m \geq 2$, by (7), we can make $m-1<f_{k}^{i}\left(S\left(H_{m}, \eta_{k}^{m}\right)<m+1\right.$. For each $i \in \mathbb{N}$, if $\bar{S} \cap H_{m} \neq \emptyset$ and $\bar{S} \cap H_{m+1} \neq \emptyset$, then $m<f_{m}^{i}(C)<m+1$;if $\bar{S} \cap H_{m} \neq \emptyset$ and $\bar{S} \cap H_{m-1} \neq \emptyset$, then $m-1<f_{m}(S)^{i}<m$; $i$.

(E) For each $\ell<j<k$ and $x \in K_{\ell}, f_{j}^{i}(x)<f_{k}^{i}(x)<f_{j}^{i}(x)+\epsilon_{j}-\epsilon_{k}$ for any

Step 2: Construction of the basic functions From (E), for any $x \in K_{m}$ and $k>m, f_{m}^{i}(x)<f_{k}^{i}(x)<f_{m}^{i}(x)+\epsilon_{1}$ for any $i=1, \ldots, 2 n+1$. Thus we can take the uniform limit of $f_{k}^{i}$ restricted on $K_{m}$. For any $x \in K_{m}$ let $\Phi_{i}(x)=\lim _{k \rightarrow \infty} f_{k}^{i}(x)$. So $\Phi_{i}$ is continuous on $K_{m}$ for each $m$. Hence $\Phi_{i}$ is continuous on $X$. Also by (D) for $x \in H_{m}, m-1<\Phi_{i}(x)<m+1+1 / 4$.

Let $\mathcal{V}_{k}^{i}=\left\{\Phi_{i}(S): S \in \mathcal{S}_{k}^{i}\right\}$. Then if $S \cap K_{m} \neq \emptyset$ and $S \in \mathcal{S}_{k}^{i}$ with $k>m$, $\Phi_{i}(S)$ is contained in the interval $\left[f_{k}^{i}(S), f_{k}^{i}(S)+\epsilon_{k}\right]$ by (E). By (B), these closed intervals are disjoint for each fixed $m$ and $k$ with $k \geq m$. Then each $\mathcal{V}_{k}^{i}$ is discrete.

Step 3: Construction of the coordinate functions Take any function $f \in C(X)$. We find $g_{1}, \ldots, g_{2 n+1} \in C(\mathbb{R})$ such that $f=\sum_{i=1}^{2 n+1} g_{i} \circ \Phi_{i}$.

For each $s \geq 0$, define the compact subset $L_{s}=K_{s+1} \backslash K_{s-1}^{\circ}$. Since $K_{1}$ is compact and $K_{1} \subseteq K_{2}^{\circ}$, there exists a function $f_{1}$ such that $f_{1}(x)=f(x)$ for $x \in K_{1}$ and $f_{1}(x)=0$ for $x \in X \backslash K_{2}^{\circ}$. Then letting $g_{1}=f-f_{1}$, it is easy to see that $g_{1}(x)=0$ for $x \in K_{1}$. Similarly, there exists $f_{2}$ such that $f_{2}(x)=g_{1}(x)$ for $x \in K_{2}$ and $f_{2}(x)=0$ for $x \in X \backslash K_{3}^{\circ}$. Inductively, $f$ can be written as an infinite sum $\sum_{s=1}^{\infty} f_{s}$ such that $f_{s}(x)=0$ for $x \in X \backslash L_{s}$.

For each $s, f_{s}$ is bounded and uniformly continuous. Fix $s \in \mathbb{N}$. Note that for each $x \in L_{s}, s-2<\Phi_{i}(x)<s+2+1 / 4$. 
By construction, if we restrict the discrete families $\mathcal{S}_{1}, \cdots, \mathcal{S}_{2 n+1}$ and the functions $\Phi_{1}, \cdots, \Phi_{2 n+1}$ to $K_{s+1}$, then the discrete families and functions are exactly those defined by Ostrand [9].

In particular, the functions $\Phi_{1}\left|L_{s}, \ldots, \Phi_{2 n+1}\right| L_{s}$ are basic for $L_{s}$ (Lemma 2). Thus we can represent $f_{s} \mid L_{s}(x)=\sum_{i=1}^{2 n+1} g_{i}^{s}\left(\Phi_{i} \mid L_{s}(x)\right)$, for some $g_{i}^{s} \in C(\mathbb{R})$. We can redefine $g_{i}^{s}$ to be constantly zero outside of $[s-2, s+2+1 / 4]$ because the image of $\Phi_{i}$ is contained in $[s-2, s+2+1 / 4]$ and $f_{s}(x)=0$ if $x \in L_{s} \backslash\left(L_{s}\right)^{\circ}$. Now $f_{s}=\sum_{i=1}^{2 n+1} g_{i}^{s} \circ \Phi_{i}$.

Finally, letting $g_{i}=\sum_{s=1}^{\infty} g_{i}^{s}$, we see that $g_{i}$ is continuous because $g_{i}(x)$ is a finite sum of non-zero continuous functions for each $x \in \mathbb{R}$, and $f=\sum_{i=1}^{2 n+1} g_{i} \circ \Phi_{i}$ - as required.

\section{Characterization of Dimension}

Lemma 10 says that a locally compact, separable metrizable space of dimension $\leq n$ has a basic family of size $\leq 2 n+1$, giving the forward implication in the 'Further' of Theorem 4, For the converse:

Lemma $13 A$ space $X$ with a basic* family $\Phi_{1}, \ldots, \Phi_{N}$, where $N \leq 2 n+1$, has dimension $\leq n$.

Proof. Take any compact subset $K$ of $X$. By Lemmal7 the maps $\Phi_{1}\left|K, \ldots, \Phi_{N}\right| K$ form a basic* family for $K$, hence by compactness a basic family. By Sternfeld's result connecting dimension and basic families in compact spaces, it follows that $\operatorname{dim} K \leq n$.

By Lemma 8, $X$ is locally compact, separable metrizable. Hence it has a locally finite cover by compact sets - each, by the above, of dimension $\leq n$. By the Locally Finite Sum Theorem for dimension, we deduce that $X$ itself must have dimension $\leq n$.

\section{References}

[1] R. Doss, A superposition theorem for unbounded continuous functions. Trans. Amer. Math. Soc. 233 (1977), 197-203

[2] Y. Hattori, Dimension and superposition of bounded continuous functions on locally compact, separable metric spaces. Topology Appl. 54 (1993), no. $1-3,123-132$

[3] D. Hilbert, Mathematische Probleme, Nachr. Akad. Wies. Gottingen (1900), 253 297, Gesammelte Abhandlungen, Bd. 3, Springer, Berlin, 1935, pp. 290-329.

[4] R. Hecht-Nielsen. Kolmogorov's mapping neural network existence theorem. In Proceedings IEEE International Conference On Neural Networks, volume II, pages 11-13, New York, 1987. IEEE Press 
[5] R. Hecht-Nielsen, Neurocomputing. Addison-Wesley, Reading, 1990.

[6] A. Kolmogorov, On the representation of continuous functions of many variables by superposition of continuous functions of one variable and addition. (Russian) Dokl. Akad. Nauk SSSR 114, 1957, pp 953-956

[7] V. Kurkova, Kolmogorov's theorem and multilayer neural networks. Neural Networks, 5:501-506, 1992.

[8] R. A. McCoy \& I. Ntantu, Topological properties of space of continuous functions, Lecture Notes in Math. 1315, Springer, 1988.

[9] P. Ostrand, Dimension of metric spaces and Hilbert's problem 13. Bull. Amer. Math. Soc. 711965 619-622

[10] B.J. Pettis, On continuity and openness of homomorphisms in topological groups. Ann. of Math. (2) 52, (1950). 293-308.

[11] Y. Sternfeld, Y Hilbert's 13th problem and dimension. Geometric aspects of functional analysis (1987-88), 1-49,Lecture Notes in Math., 1376, Springer, Berlin, 1989. 